\title{
Compliance with Screening and Treatment of Latent Tuberculosis among Health Care Workers in a Lebanese Hospital
}

\author{
Stephanie Farah ${ }^{\text {a\# }}$, Youssef Rizk ${ }^{\text {b\# }}$, Georges Khazen ${ }^{c}$, Rania Sakr ${ }^{\text {d }}$ \\ ${ }^{a}$ Lebanese American University Medical Center -Rizk Hospital, Department of Endocrinology, Beirut, Lebanon; \\ stephanie.faresfarah@lau.edu \\ ${ }^{\mathrm{b}}$ American University of Beirut Medical Center, Department of Family Medicine, Beirut, Lebanon; \\ youssef.rizk90@gmail.com \\ ${ }^{\mathrm{c}}$ Lebanese American University, Department of Computer Science and Mathematics; gkhazen@lau.edu.lb \\ ${ }^{\mathrm{d}}$ Lebanese American University Medical Center -Rizk Hospital, Department of Family Med-icine, Beirut, Lebanon; \\ raniasakr@hotmail.com
}

*Corresponding Author: Rania Sakr; Lebanese American University Medical Center-Rizk Hospital, Department of Family Medicine, Beirut, Lebanon; rani-asakr@hotmail.com

${ }^{\#}$ These Authors have contributed equally to this manuscript

Received 13 August 2021;

Accepted 28 August 2021;

Published 02 September 2021

\begin{abstract}
Background: Health care workers (HCWs) are at increased risk of Tuberculosis infection. Various Guidelines recommend pre-placement, postexposure, and annual screening for latent tuberculosis among HCWs. $\underline{\text { Aim: }}$ to assess the compliance of HCWs with these protocols. In addition, the study aimed to evaluate the compliance with treatment of Latent Tuberculosis and reasons of non-adherence. Methods: Study was conducted on $560 \mathrm{HCWs}$ in a Lebanese hospital. A Questionnaire collected data on knowledge about latent tuberculosis, post-exposure screening and the reasons behind non adherence to treatment. A retrospective chart review on the same HCWs revealed data regarding TST preemployment status, compliance to screening, seroconversion and compliance to treatment. Results: $69 \%$ of HCWs did not attend a lecture on Tuberculosis. $76 \%$ were aware of screening policies. $88 \%$ performed pre-placement testing. $29 \%$ were screened post exposure. Only $4.3 \%$ had their PPD done annually. $8 \mathrm{HCWs}$ seroconverted; Only $59 \%$ of positive employees initiated treatment. . Reasons included refusal or belief of no treatment benefit (55\%), not being prescribed a treatment (33\%), Contraindications (8\%), and being afraid of side effects $(4 \%)$. 15\% of those who received treatment were not able to complete the full course mainly due to side effects (80\%). Conclusion: We reported acceptable rates of pre-placement screening, low rates of annual screening and post exposure screening. We also reported fair rates of initiation and completion of LTBI treatment. We recommend an administrative initiative to strongly implement the policies of screening for LTBI.
\end{abstract}

Keywords: Healthcare Workers, Latent Tuberculosis Infection, Compliance, Screening, Treatment.

\section{Introduction}

Tuberculosis (TB) remains one of the world's deadliest communicable diseases. In 2013, an estimated 9.0 million people developed TB and 1.5 million died from the disease world-wide ${ }^{[1]}$. The majority of cases occur in developing countries. In Lebanon, the incidence rate of active TB in 2017 was $12 / 100000$ population and the mortality rate was around $0.95 / 100000$ population, making Lebanon a low TB burden country ${ }^{[2]}$.
HCWs are at increased risk of exposure to TB infection as a result of the high level of occupational contact with infected individuals ${ }^{[3]}$. TB is also more prevalent among health care workers (HCWs) as compared to the general population ${ }^{[4]}$. Therefore, screening for Latent TB infection is very important in this group.

According to 2019 Center for Disease Control and Prevention (CDC) guidelines, pre- placement screening is recommended for all health care personnel. Serial screening is also routinely recommended for $\mathrm{HCWs}$ who reside in a high $\mathrm{TB}$ incidence rate country (any country other than Australia, Canada, 
US, Western or northern Europe and New Zealand); or HCWs who are immunocompromised ${ }^{[5]}$. In addition, the world health organization (WHO) recommends that $\mathrm{HCWs}$ with baseline negative test results, should receive TB screening annually ${ }^{[6]}$.

Approximately 5\%- $10 \%$ of HCWs infected and not treated for Latent tuberculosis infection (LTBI) will develop active disease ${ }^{[4]}$. The risk of progression of LTBI, defined as the presence of mycobacterium TB bacteria without the manifestation of signs or symptoms, to TB disease is highest during the first years after infection ${ }^{[4]}$. Several studies have shown that HCWs are not compliant with screening and/or preventive measures ${ }^{[7,8,9]}$. Such data have led to a growing interest in improving the detection and proper management of TB infection among HCWs. To our knowledge, no studies so far have examined the compliance of HCWs with screening and treatment protocols of LTBI in Lebanon.

Therefore, the primary outcome was set to be the evaluation of the compliance of health care workers at a Lebanese University Medical Center with performing pre-placement TB screening, post exposure screening and annual TB screening. The secondary objectives are to evaluate the compliance of HCWs diagnosed with LTBI with the treatment protocols and reasons of non-adherence to treatment.

\section{Methods}

The current study was conducted at the Lebanese American University Medical Center - Rizk Hospital (LAUMC-RH), an urban, tertiary care hospital of 100 beds located in Beirut, Lebanon from 2016 to 2017. $560 \mathrm{HCWs}$ (medical, nursing, and allied health professionals) were serving at the hospital in 2016; The study excluded attending physicians, resident physicians, and medical students, because of lack of documented data.

We conducted a cross-sectional study through both a prospective evaluation via closed ended questionnaires and a retrospective chart review using clinical records of HCWs working at LAUMC-RH. HCWs included in the study were healthcare assistants and logistic staff in the following departments: Laundry, admission offices, maintenance, outpatient clinics, physiotherapy department, neurophysiology department, nutrition department, pharmacy, pathology department, sterilization department, and kitchen. The aim of the questionnaire was to collect data on socio demographic characteristics of the participants, scope of practice, du-ration of work, knowledge about latent tuberculosis effects and policies, vaccination status, previous contact with positive Tuberculosis cases, and frequency of post-exposure screening. In addition, we collected data on the reasons behind refusing or discontinuing treatment.

After writing the questionnaire in English, it was translated to Arabic. We then back-translated it to English to ensure the accuracy of the translation. The Arabic and English versions were consistent. A pilot test was conducted on a sample of 20 respondents in both languages. The purpose was to know if there is confusion about any items, test for feasibility, and whether respondents have suggestions for possible improvements of the items. The questionnaire was composed of 26 questions needing a period of no more than 10 minutes to be completed. It was conducted in both English and Arabic, according to the preference of the employee. A single researcher (physician) was dedicated in order to explain the nature of the study and to clarify any inquiry the employee had.

An informed consent was obtained from the employees prior to filling the questionnaire. In parallel, a retrospective chart review of medical records was conducted on the same employees that had completed the questionnaires. The purpose of the chart review was to gather data regarding the purified protein derivative (PPD), pre placement status, compliance to annual two-step testing (TST) screening, change in TST status during employment, and compliance to treatment protocols and duration.

All surveys and chart reviews were completed within 1 year (2018).

Institutional review board (IRB) approval was obtained before beginning this study at the Lebanese American University.

The following definitions were adapted: Pre-placement TST screening test: Two-step testing by administering first TST following proper protocol and documenting the result after 48-72 hours followed by Retesting in 1-3 weeks.

Post-exposure screening: Health care personnel with a previous negative TB test result should be tested immediately and re-tested 8 to 10 weeks after the last known exposure.

Positive skin test: induration of $10 \mathrm{~mm}$ or greater following a TST.

TST seroconversion: $\geq 10 \mathrm{~mm}$ increase in the size of the TST induration with a documented negative $(<10 \mathrm{~mm})$ baseline twostep TST result ${ }^{[10]}$.

The R statistical program was used for all statistical analysis. The chi-square and Fisher exact tests were used to compare the variables. The chi-square test was used to assess the associated of the gender and age group with the history of TB, pre-placement status and PPD testing. We also checked whether there is a significant relationship between the previous occupation and current work department with the history of TB and pre-placement status respectively. A significant association is considered if the $\mathrm{p}$ value is $<0.05$ after applying a Bonferroi correction for multiple testing.

\section{Results}

Table 1 summarizes the socio demographic characteristics of the Healthcare workers included in the study. In total, 560 healthcare workers were included; $36 \%$ were males (202) and $64 \%$ were females (358). None of the approached healthcare workers refused to participate. $38 \%$ (215) of these workers were previously employed in a healthcare setting before joining the current institution. Of these, $27 \%$ (151) were in the nursing field. $69 \%$ (384) of the workers were currently involved in direct patient care. Employees having direct contact with patients were working in the following units: Regular floors, ICU, ER, dialysis, endoscopy, operation room, private clinic, dermatology, ophthalmology, radiology, laboratory, physiotherapy, neurophysiology, and nutrition. The remaining staff, $31 \%$ (176), had minimal contact with patients, such as those working in the Administration, pharmacy, pathology, sterilization department, and kitchen. $42 \%$ (233) of healthcare workers have been working for more than 15 years at LAUMCRH.

When asked about their awareness about Tuberculosis disease, most of the healthcare workers $69 \%$ (386) reported that they did not attend a lecture, seminar or workshop on Tuberculosis. In addition, when asked about their awareness regarding policies of TB screening in a healthcare setting, only $49 \%$ (274) said that they were aware of such policies. The major-ity of the HCWs $76 \%$ 
(426) reported that they were aware of all types of policies including screening, precautions, and treatment.

Most of them were not BCG vaccinated 65\% (365); while only $13 \%$ (75) received the vaccination. The remaining ones $21.42 \%$ (120) said that they do not recall their immunization status. $26 \%$ (143) reported a history of contact with a confirmed TB case. The majority of them were previously working in a healthcare setting ( $p$ value 3.82-08) (Table 2). Of those who re-ported exposure to TB, only 29\% (42/143) were screened post exposure. Most of them were between the ages of 30 and 50 (P value 0.04). In our hospital, pre-placement testing was performed on $88 \%$ (493) of healthcare workers, out of which 50 positive cases were detected. There was a significant association between preplacement testing and age group with the majority being between $30-50$ ( $\mathrm{p}$ value 0.0008 ) (Table 3 ).

After employment, only $61 \%$ (344) of healthcare workers had PPD tests performed through-out the years of employment, which was also significantly associated with age group, and the majority being between 30 and 50(p value 1.66-09).

We found that only $4.2 \%$ (24) of the HCWs had their PPD done annually (Table 4). A total of 8 HCWs seroconverted; out of which 3 seroconverted within 5 years of employment, one within 9 years, and the remaining within more than 15 years of employment. Table 5 specifies the departments in which the seroconverted HCWs work.

Only 34 out of the 58 (50 at pre-placement and 8 seroconverted) PPD positive patients received treatment. 94\% (32) of those treated received Isoniazid regimen for 6-9 months; $6 \%$ (2) received a combination of both Isoniazid and Rifampin.41\% (24) did not receive any treatment. The most common reasons were their belief of no treatment benefits/refusal of treatment 55\% (13), not given a prescription $33 \%$ (8), contraindications $8 \%$ (2), and afraid of side effects $4 \%$ (1). Of those who received treatment, $15 \%$ (5) were not able to complete the course mainly due to side effects $(4 / 5)$ or pregnancy $(1 / 5)$.

Table 1: Socio demographic Profile

\begin{tabular}{|l|l|l|}
\hline \multicolumn{2}{|l|}{ Mrequency (\%) } \\
\hline Gender & Male & $202(36)$ \\
& Female & $358(64)$ \\
\hline Age (years) & Less or equal to 30 & $129(23)$ \\
& $30-50$ & $335(60)$ \\
& More than 50 & $96(17)$ \\
\hline Education & High School Degree & $217(39)$ \\
& College Degree & $263(47)$ \\
& Post Graduate Degree & $63(11)$ \\
\hline Previous Occupation before getting hired at & No degree awarded & $17(3)$ \\
\hline current institution. & HCWs in nursing Field & $151(27)$ \\
& HCWs in non-Nursing Field & $64(11)$ \\
\hline Current Occupation at LAUMCRH & Non-healthcare Occupation & $345(62)$ \\
\hline Duration of Work at LAUMCRH (years) & Direct Contact with patients & $384(69)$ \\
& Minimal/no contact with patients & $176(31)$ \\
\hline
\end{tabular}

Table 2: History of TB contact as a function of Gender, Age Group (years) and Previous Occupation

\begin{tabular}{|l|l|l|l|}
\hline History of TB Contact & Yes (\%) & No (\%) & Adjusted P value \\
\hline Gender & & & \\
Male & $56(39)$ & $146(35)$ & \\
Female & $87(61)$ & $271(65)$ & 0.43 \\
\hline Total & 143 & 417 & \\
\hline Age & & & \\
Less or equal to 30 & $41(29)$ & $88(21)$ & \\
$30-50$ & $82(57)$ & $253(61)$ & \\
More than 50 & $20(14)$ & $76(18)$ & $\mathbf{0 . 1 4}$ \\
\hline Total & $\mathbf{1 4 3}$ & $\mathbf{4 1 7}$ & \\
\hline Previous healthcare Occupation & & & $132(32)$ \\
Yes & $83(58)$ & $285(68)$ & $\mathbf{3 . 8 2}^{-\mathbf{0 8}}$ \\
No & $60(42)$ & $\mathbf{4 1 7}$ & \\
\hline Total & $\mathbf{1 4 3}$ & & \\
\hline
\end{tabular}

Table 3: Pre-placement status by Gender, Age Group (years), and Current Work de-partment

\begin{tabular}{|l|l|l|l|l|}
\hline Pre-placement status & Positive (\%) & Negative (\%) & Not performed (\%) & Adjusted P value \\
\hline Gender & & & & \\
\hline Male & $22(44)$ & $162(37)$ & $18(27)$ & \\
Female & $28(56)$ & $281(63)$ & $49(73)$ & \\
\hline Total & $\mathbf{5 0}$ & $\mathbf{4 4 3}$ & $\mathbf{6 7}$ & $\mathbf{0 . 1 4}$ \\
\hline Age & & & & \\
\hline
\end{tabular}




\begin{tabular}{|l|l|l|l|l|}
\hline Less or equal to 30 & $10(20)$ & $113(26)$ & $6(9)$ & \\
$30-50$ & $28(56)$ & $267(60)$ & $40(60)$ & \\
More than 50 & $12(24)$ & $63(14)$ & $21(31)$ & $\mathbf{0 . 0 0 0 8}$ \\
\hline Total & $\mathbf{5 0}$ & $\mathbf{4 4 3}$ & $\mathbf{6 7}$ & \\
\hline Current Work department & & & & \\
Direct contact with patients & $36(72)$ & $304(69)$ & $44(66)$ & \\
Minimal or no contact with patients & $14(28)$ & $139(31)$ & $23(34)$ & $\mathbf{0 . 7 7}$ \\
\hline Total & $\mathbf{5 0}$ & $\mathbf{4 4 3}$ & $\mathbf{6 7}$ & \\
\hline
\end{tabular}

Table 4: Frequency of PPD testing after employment by Gender and Age Group (years)

\begin{tabular}{|l|l|l|l|}
\hline Frequency of PPD testing after employment & Annually (\%) & Randomly (\%) & Adjusted P value \\
\hline Gender & & & \\
\hline Male & $13(54)$ & $102(32)$ & \\
Female & $11(46)$ & $218(68)$ & \\
\hline Total & $\mathbf{2 4}$ & $\mathbf{3 2 0}$ & $\mathbf{0 . 0 4}$ \\
\hline Age & & & \\
\hline Less or equal to 30 & $16(67)$ & $33(10)$ & \\
$30-50$ & $7(29)$ & $219(68)$ & \\
More than 50 & $1(4)$ & $68(22)$ & \\
\hline Total & $\mathbf{2 4}$ & $\mathbf{3 2 0}$ & $\mathbf{1 . 6 6}^{-\mathbf{0 9}}$ \\
\hline
\end{tabular}

Table 5: Divisions where HCWs who seroconverted work and duration of employment at the time of seroconversion.

\begin{tabular}{|l|l|}
\hline Divisions & Duration of employment (years) \\
\hline Laboratory/blood bank technician & 5 \\
\hline Billing Assistant & 15 \\
\hline Registered nurse floor & 2 \\
\hline Orderly (Radiology) & 3 \\
\hline Registered nurse ( wound Care) & 28 \\
\hline Practical nurse( ICU) & 20 \\
\hline Practical nurse (Oncology) & 9 \\
\hline Registered nurse( one Day Unit) & 17 \\
\hline
\end{tabular}

\section{Discussion}

The frequency of LTBI in our hospital was $10.4 \%$ (58), comparable to published findings in the same geographic region. A study conducted in four major tertiary care hospitals in Saudi Arabia that showed an overall prevalence of $11 \%{ }^{[11]}$. Additionally, Balkhi et al showed a low rate of pre-placement TST testing of approximately $40 \%$ in a tertiary hospital in Saudi Arabia ${ }^{[12]}$. We reported an acceptable rate $88 \%$ of latent tuberculosis preplacement testing. This result is superior to that reported by Driver et al. where unknown TST status at pre-placement was approximately $21 \%$ among HCWs in New York ${ }^{[13]}$. Our result is also superior to results reported from similar studies in the Region. 143 HCWs reported exposure to TB during their career. The post exposure screening rate was only $29 \%$ (42). This is a very low rate, especially that the CDC guidelines are very assertive that post exposure screening is highly recommended. This emphasizes the need of administrative enforcement of these policies with closer follow up.

In 2004, Salpeter et al have shown that a 1-year screening interval is a cost effective and safe choice at low, moderate, and high Risk for Tuberculosis Exposure ${ }^{[14]}$. However, only $4.3 \%$ in our hospital performed annual TST screening and most of them were younger than 30 years old (p value 1.66-09). We recommend more emphasis on implementation of annual screening by the occupational medicine team at the hospital to prevent future infection burden and treatment costs.

Seroconversion was witnessed in $8 \mathrm{HCWs}$; half of them seroconverted within the first 10 years of employment, while the other half seroconverted between 10 and 30 years. 63\% (353) were working in the nursing field. This rate is comparable to the results found in a study done in Italy where $65.7 \%$ of the exposed HCWs were nurses and $77.8 \%$ who seroconverted were nurses ${ }^{[15]}$.

LTBI therapy has proven to decrease disease progression to active TB by $60-90 \%{ }^{[16]}$. Despite this proven effectiveness, studies have demonstrated that those with LTBI often de-cline treatment, and many do not complete it ${ }^{[12]}$. We reported a fair rate of treatment initiation of 58.6\% (34). This rate was lower compared to the rate found in a study done in Riyadh, that showed that $93 \%$ of the HCWs who tested positive started treatment ${ }^{[12]}$. However, we reported high rates of completion of the full course of treatment (69 months) reaching $85.3 \%$ (29). In comparison, Balkhi et al showed that only $17.4 \%$ completed the 9 -month course in a tertiary hospital in Riyadh ${ }^{[12]}$. Another study done at 32 U.S. and Canadian medical clinics showed that only $47.6 \%$ completed the full course (6-9 month) treatment ${ }^{[8]}$. A previous study has shown that several factors have been linked to non-compliance to treatment including lack of follow-up, physician advice to discontinue therapy because of peripheral neuropathy, pregnancy, or inappropriate prescription ${ }^{[12]}$. Other factors include longer duration of treatment course, long waiting time for appointment, inconvenient office hours, and complicated telephone system ${ }^{[17]}$. However, in our study, failure to complete the recommended 6-9 months course was mainly due to side effects (4) and new Pregnancy (1). Rennie et al demonstrated that offering a shorter choice of regimen improves completion. Most patients chose the 3-month rifampicin and isoniazid over the 6-month isoniazid treatment ${ }^{[18]}$. Joseph et al reported that misconceptions such as the belief that TB was hereditary or a blood infection and that transmission could occur through handling writing utensils or office folders, emerged frequently in routine 
TST and LTBI treatment non adherent groups ${ }^{[7]}$. In our findings, $41.4 \%$ (24) Latent TB positive HCWs did not initiate any therapy. The most common reasons were their belief of no treatment benefits/refusal of treatment 55\% (13), not given a prescription $33 \%$ (8), contraindications $8 \%$ (2), afraid of side effects $4 \%$ (1). Only $49 \%$ re-ported that they were aware about policies concerning tuberculosis screening and that only $31 \%$ attended a lecture/seminar/ workshop on Tuberculosis screening. We suggest that more awareness should be made in the hospital through annual lectures or workshops regarding policies, benefits from preventing active $\mathrm{TB}$, and the consequences from not adhering to treatment. We consider that this might improve the compliance to screening and treatment.

We acknowledge some limitations in our study. First, we excluded all doctors and medical students due to lack of proper documentation of data in their health records despite them forming a substantial proportion of the health care workers staff. Second, we admit that there might be recall bias from interviewing the HCWs. Third, being a single-center study may limit the generalizability of our findings. Despite these limitations, we believe that documenting these deficiencies may be helpful to improve tuberculosis control measures at our hospital and other hospitals in the region with similar challenges.

\section{Conclusion}

In conclusion, we reported acceptable rates of pre-placement screening, low rates of annual screening and post exposure screening for TB among HCWs. In addition, we reported fair rates of initiation and completion of LTBI therapy among positively documented cases at our hospital. We recommend an administrative initiative at the hospital to more strongly implement the policies of screening for LTBI. This can be achieved by closer follow up on annual screening of all HCWs at the hospital, improved documentation of statuses, including physicians, and medical students, enforcing evaluation of all HCWs following exposure to an infectious source, encouraging the initiation of shorter courses of LTBI therapy, and increasing education through regular workshops and seminars targeting HCWs in the institution.

\section{Acknowledgement}

Not applicable.

\section{Financial support}

Not applicable.

\section{Conflict of interest}

None

\section{References}

[1] World Health Organization. WHO report 2014. Global tuberculosis control: epidemiology, strategy, financing. WHO/HTM/ TB/2014.411. Geneva, Switzerland: WHO, 2014.

[2] Ministry of public health. National TB program annual report-2018. Current health event. Volume 6, Issue $03-$ March 2019. https://applications.emro.who.int/docs/leb/2019/Epi_Mo nitor_2019_6_03.pdf?ua=1\&ua=1
[3] Louther J, Riviera P, Feldman J, et al. Risk of tuberculin conversion according to occupation among health care workers at a New York City hospital. Am J Respir Crit Care Med 1997; 156: 201-205.

[4] Jensen P A, Lambert L A, Iademarco M F, Ridzon R; Centers for Disease Control and Prevention. Guidelines for preventing the transmission of Mycobacterium tuberculosis in health-care settings, 2005. MMWR Recomm Rep 2005; 54 (RR-17): 1- 141.

[5] Sosa LE., et al. Tuberculosis Screening, Testing and Treatment of US Health Care Personnel: Recommendations from the National Tuberculosis Controllers Association and CDC, 2019. MMWR Morb Mortal Wkly Rep 2019;68:439-443.

[6] World health organization. TB infection control in health care facilities, congregate settings, and households, <http://www.who.int/tb/health_systems/en/o $\mathrm{n}>$; 2012 [accesed 1.30.2016].

[7] Joseph H, Kuwahara R, Lowry D, Lambert L, Panlilio A, Raucher B, Holcombe J, Poujade J, Rasmussen D, Wilce M. Factors influencing health care workers' ad-herence to work site tuberculosis screening and treatment policies.Am J Infect Control. 2004 December; 32(8): 456-461. doi: 10.1016/S0196655304004791

[8] Horsburgh, CR. Latent TB Infection Treatment Acceptance and Completion in the United States and Canada. Chest. 2010;137(2):401-409.

[9] Taubman D, Titler N, Edelstein H, Elias M, Saliba W. Providing detailed information about latent tuberculosis and compliance with the PPD test among healthcare workers in Israel: a randomized controlled study. J Epidemiol Glob Health. 2013 Dec;3(4):253-60. doi: 10.1016/j.jegh.2013.06.003

[10] Jensen P. Guidelines for Preventing the Transmission of Mycobacterium tuberculo-sis in Health-Care Settings, $2005 . \quad$ Cdc.gov. https://www.cdc.gov/mmwr/preview/mmwrhtml/rr5417a 1.htm. Published 2019. Accessed March 11, 2019.

[11] Abbas MA, AlHamdan NA, Fiala LA, AlEnezy AK, AlQahtani MS. Prevalence of latent TB among health care workers in four major tertiary care hospitals in Riyadh, Saudi Arabia. J Egypt Public Health Assoc. 2010;85(1-2):61-71.

[12] Balkhy HH, Miller TL, Ali S, Nuzzo JB, Kentenyants K, El-Saed A, McNabb SJ. Compliance with postexposure screening and treatment of latent tuberculosis in-fection among healthcare workers in a tertiary care hospital in Saudi Arabia. Infect Control Hosp Epidemiol. 2014 Feb;35(2):176-81. doi: 10.1086/674855

[13] Driver, C.R. et al, Tuberculosis in health care workers during declining tuberculosis incidence in New York State. American Journal of Infection Control, 2015; 33 (9), pp. 519-526.

[14] Salpeter S, Salpeter E, Screening and treatment of latent tuberculosis among healthcare workers at low, moderate, and high risk for tuberculosis exposure: a costeffectiveness analysis. Infect Control Hosp Epidemiol. 2004 December; 25(12): 1056-1061. doi: $10.1086 / 502343$

[15] Muzzi A, Seminari E, Feletti T, et al. Post-exposure rate of tuberculosis infection among health care workers measured with tuberculin skin test conversion after unprotected exposure to patients with pulmonary 
tuberculosis: 6-year experience in an Italian teaching hospital. BMC Infect Dis. 2014;14:324. Published 2014 Jun 12. doi:10.1186/1471-2334-14-324

[16] Lobue P, Menzies D. Treatment of latent tuberculosis infection: an up-date. Respirology. 2010:15(4);603-22. 10.1111/j.1440-1843.2010.01751.

[17] Treatment of Latent TB Infection | LTBI: A Guide for Primary Health Care Provid-ers| Guides \& Toolkits |
Publications \& Products | TB | CDC. Cdc.gov. https://www.cdc.gov/tb/publications/ltbi/treatment.htm. Published 2019. Accessed March 14, 2019.

[18] Rennie TW., et al. Patient choice promotes adherence in preventive treatment for latent tuberculosis. Eur Respir J 2007; 30(4): 728-735 